\title{
Intercomprensione e plurilinguismo: studenti universitari di lingue e di discipline non linguistiche a confronto
}

\author{
Fabiana Rosi ${ }^{1}$
}

Ricevuto: 20 novembre 2019 / Modificato: 25 maggio 2020 / Accettato: 30 giugno 2020

Riassunto: La ricerca confronta la capacità di intercomprensione dello spagnolo fra due gruppi di italofoni nativi, studenti universitari di lingue straniere e studenti universitari di discipline non linguistiche, prevalentemente di ingegneria, per verificare se la competenza linguistica plurilingue e la competenza metalinguistica più matura degli studenti di lingue determinino un vantaggio nella comprensione di un testo scritto in una lingua affine non conosciuta. Le analisi evidenziano analogie piuttosto che differenze fra i due gruppi sia sul piano della comprensione del contenuto, misurata in termini di comprensione globale, analitica e inferenziale, sia delle strategie di comprensione adottate. Il vantaggio degli studenti di lingue emerge solo nella rapidità di trasposizione del testo spagnolo in italiano. I risultati sottolineano così come l'intercomprensione fra lingue affini sia un fenomeno altamente spontaneo e incoraggiano ad impiegarla come risorsa efficace per incrementare la motivazione all'apprendimento di nuove lingue e promuovere il plurilinguismo.

Parole chiave: intercomprensione; lingue affini; competenza metalinguistica; plurilinguismo; motivazione; transfer; falsi amici.

\section{[en] Intercomprehension and plurilingualism: A comparison between university students of languages and students of non-linguistic disciplines}

\begin{abstract}
The present study compares competence in the intercomprehension of Spanish by two groups of Italian native speakers: students of foreign languages and students of non-linguistic disciplines, mainly engineering, at university level. The aim is to verify whether the plurilingual competence and the more developed metalinguistic competence of students of languages facilitate the understanding of a text written in an unknown cognate language. The analyses show similarities more than differences between the two groups in the comprehension of content, which is assessed in terms of global, analytic and inferential comprehension, as well as in comprehension strategies. The advantage of students of languages emerges only in the amount of time they needed for translating the text from Spanish into Italian. The findings confirm that intercomprehension among cognate languages is a highly spontaneous phenomenon and boost the usage of intercomprehension as an effective resource to increase motivation in language learning and to promote plurilingualism.
\end{abstract}

Keywords: intercomprehension; cognate languages; metalinguistic competence; plurilingualism; motivation; transfer; false friends.

Sommario: Introduzione 1. Principi e modelli di intercomprensione 1.1. L'intercomprensione come processo 1.2. L'intercomprensione fra spagnolo e italiano 1.3. Le applicazioni didattiche dell'intercomprensione 2. Un esperimento di intercomprensione 2.1. Partecipanti 2.2. Metodologia 3. Analisi

Università di Trento, via Tommaso Gar 14, 38122 Trento

E-mail: fabiana.rosi@unitn.it 
3.1. La comprensione del testo 3.2. La trasposizione del testo in L1 3.2.1. Accuratezza lessicale 3.2.2. Accuratezza morfologica 3.2.3. Accuratezza sintattica 3.3. Strategie di comprensione 4. Osservazioni conclusive.

Come citare: Rosi, Fabiana (2020): «Intercomprensione e plurilinguismo: studenti universitari di lingue e di discipline non linguistiche a confronto», Cuadernos de Filología Italiana, 27, pp. 137-165.

\section{Introduzione}

La capacità di comprendere parzialmente una lingua non conosciuta ma affine ad una delle lingue appartenenti al repertorio individuale del parlante, definita intercomprensione (Blanche-Benveniste / Valli 1997; Benucci 2005, 2015; De Carlo 2011; Bonvino / Jamet 2016), è un fenomeno di comunicazione plurilingue spontaneo attestato fin dall'antichità (Aslanov 2016). Si tratta di un'operazione di ricostruzione linguistica da parte del parlante, che elabora induttivamente una grammatica comparativa (Simone 1997) grazie all'osservazione della nuova lingua e al confronto fra questa e le lingue a lui già note, al fine di individuare analogie interlinguistiche che lo aiutino a riconoscere i primi elementi della nuova lingua e ad accedere gradualmente al significato. Centrali nel processo di intercomprensione risultano dunque la conoscenza di più lingue e la competenza metalinguistica, in quanto la capacità di riflettere sulla lingua permette di individuarne le sistematicità ed estrapolare le regole d'uso degli elementi linguistici che la costituiscono (Berretta 1984; Lo Duca 2003, 2004, 2012).

Nella presente ricerca si mettono a confronto studenti universitari del secondo anno di studi magistrali, caratterizzati da differenti conoscenze linguistiche e da un diverso grado di maturazione di competenza metalinguistica. Il primo gruppo è composto da studenti universitari di lingue straniere, che conoscono approfonditamente più di una lingua e sono impegnati costantemente in attività grammaticali e traduttive per potenziare la propria competenza linguistica e metalinguistica nelle diverse lingue studiate (Bialystok 1988; Camps / Milian 1999). Tali studenti nei cinque anni del percorso universitario hanno potuto sviluppare un alto grado di competenza linguistica in più lingue, costruendosi un ricco repertorio plurilingue, di competenza metalinguistica implicita ${ }^{2}$, attraverso l'impiego di tali lingue nella propria pratica linguistica pressoché quotidiana, e di competenza metalinguistica esplicita, grazie allo studio della grammatica e alle attività didattiche focalizzate sulla riflessione sulla lingua nei corsi frequentati all'università. Il secondo gruppo, invece, è composto da studenti universitari di discipline non linguistiche, prevalentemente di ingegneria. La loro competenza linguistica è ugualmente plurilingue, grazie allo studio di almeno una lingua straniera nel percorso scolastico preuniversitario, ma i livelli di padronanza linguistica raggiunti sono meno avanzati. Inoltre, a causa delle ridotte comunicazioni in lingue diverse dall'italiano e della minor consuetudine a riflettere sulla lingua negli anni dello studio accademico, la loro competenza metalinguistica ha raggiunto un livello di maturazione inferiore rispetto ai colleghi del corso di studi

\footnotetext{
La complessa natura della competenza metalinguistica può essere suddivisa in competenza metalinguistica implicita ed esplicita in base al livello di consapevolezza del parlante e ad altri criteri (Ellis 2004, 2005, 2006; Roehr 2007), come discusso nel par. 1.
} 
in lingue. Per quanto riguarda le altre variabili pertinenti, i due gruppi sono comparabili, condividendo L1, età, livello di istruzione, assenza di conoscenze pregresse nello spagnolo e nelle tecniche di intercomprensione. Scopo delle analisi è verificare se un livello superiore di competenza linguistica e metalinguistica porti ad un effettivo vantaggio nelle capacità di intercomprensione, nell'ambito della ben nota facilità di intercomprensione fra spagnolo e italiano (Calvi 1995, 2001; Cortés Velásquez 2016; Di Gesù 2016). Le capacità di intercomprensione vengono rilevate mediante domande di comprensione globale, analitica e inferenziale di un testo spagnolo scritto, la trasposizione del testo in italiano e le riflessioni degli studenti sulle strategie adoperate per risolvere tali compiti. Le analisi si concentrano pertanto non solo sulla correttezza e accuratezza delle risposte fornite nell'attività di intercomprensione, ma anche sui processi messi in campo dai partecipanti per produrre tali risposte sulla base, da un lato, delle autodichiarazioni degli studenti sulle difficoltà incontrate $\mathrm{e}$ sulle strategie impiegate per superarle, dall'altro, dell'analisi distinta per le diverse tipologie di errore emerse nei dati, che segnalano differenti approcci al testo da parte del parlante.

L'articolo è strutturato come segue: il paragrafo 1 inquadra il tema dell'intercomprensione dal punto di vista teorico generale, specifico per l'italiano e lo spagnolo ed applicativo; il paragrafo 2 presenta il campione intervistato e la metodologia di raccolta dati; il paragrafo 3 illustra le tendenze emerse per quanto riguarda la comprensione del testo spagnolo, la sua trasposizione in italiano e le strategie adottate dagli studenti durante tali compiti. Nelle osservazioni conclusive, alla luce dei risultati sono discusse implicazioni didattiche e istituzionali sull'impiego dell'intercomprensione come risorsa efficace nell'offerta di formazione linguistica all'università e nella promozione del plurilinguismo.

\section{Principi e modelli di intercomprensione}

\subsection{L'intercomprensione come processo}

I meccanismi alla base del processo di intercomprensione sono delineati da numerosi studi (Blanche-Benveniste / Valli 1997; Bonvino / Jamet 2016): il parlante osserva per la prima volta una lingua nuova; confrontandola con le lingue note pian piano riconosce analogie fra questa e le lingue appartenenti al suo repertorio personale; ricostruisce comparativamente il significato di singole parole, elementi morfologici, strutture sintattiche ed aspetti pragmatici; arriva così, in modo graduale, a formarsi induttivamente una grammatica e un lessico della nuova lingua. La riflessione sulla lingua necessaria per intercomprendere, dunque, si sviluppa a livello interlinguistico: la lingua target non ancora conosciuta viene analizzata grazie al confronto con la L1 e con le L2 note al parlante. Per questo, più è ricco il repertorio linguistico del parlante, considerando tutto il materiale linguistico a sua disposizione (madrelingua, dialetti, lingue studiate in precedenza, lingue conosciute anche limitatamente), più è ampia la gamma di osservazioni e scoperte che il parlante può fare.

Come evidente, il confronto interlinguistico alla base dell'intercomprensione è reso possibile sia dalla competenza linguistica plurilingue sia dalla competenza metalinguistica, la capacità di riflettere sulla lingua, di tipo sia implicito che esplicito. 
La distinzione fra i due tipi di competenza metalinguistica non è univoca, come non sono univoci i termini adottati nei vari studi per definire tali costrutti. Seguendo la tassonomia di Rod Ellis $(2004,2005,2006)$, la competenza metalinguistica implicita è più inconscia; permette ad esempio al parlante di individuare frasi agrammaticali, ma non di spiegare la causa dell'agrammaticalità; è procedurale, ovvero permette la rapida elaborazione e applicazione delle regole grammaticali in vista della comunicazione; è più solida e sistematica; è più automatica; è accessibile anche in condizioni di comunicazione on-line dai tempi serrati; non è verbalizzabile. La competenza metalinguistica esplicita, dall'altra parte, è più conscia, permette, ad esempio, di motivare i giudizi di grammaticalità dati dal parlante; è di tipo dichiarativo, in quanto è immagazzinata sotto forma di regola; è meno stabile nella memoria del parlante; richiede un maggior controllo cognitivo del parlante per essere espressa; richiede condizioni comunicative dai tempi più rilassati; è verbalizzabile. La competenza metalinguistica implicita è spontanea e naturale, tutti i parlanti sono in grado nei primi anni di vita di riflettere sulla propria madrelingua (L1), di coglierne le sistematicità e ricostruirne la grammatica senza studiarla (Voghera 2008), mentre la competenza metalinguistica esplicita si forma con lo studio delle lingue e della grammatica. È bene precisare comunque che i due tipi di competenza metalinguistica, pur essendo distinti da specifiche peculiarità, non sono divisi in maniera netta, bensì si configurano come i due estremi di un continuum e sono misurabili con strumenti diagnostici scalari e progressivi sviluppati ad hoc, come i test di abilità metalinguistiche TAM del progetto MATEL (Pinto 2015).

La ricostruzione comparativa alla base dell'intercomprensione è favorita dall'affinità fra la lingua target e le lingue note al parlante: la prossimità strutturale fra le lingue innesca il fenomeno del transfer (Gass / Selinker 1983; Odlin 1989; Jarvis / Pavlenko 2008), agevolando il trasferimento degli elementi linguistici da una lingua all'altra. Secondo il principio «transfer-to-somewhere» di Andersen (1983), un elemento linguistico è trasferito fra due lingue più facilmente quando queste sono percepite dal parlante come simili fra loro, in quanto sono numerosi i casi in cui l'uso di elementi già noti da altre lingue risulta efficace a livello comunicativo anche nella lingua target e ciò induce il parlante ad attingere alle lingue note con frequenza e fiducia di successo comunicativo. Com'è noto, il transfer può avere esito comunicativo efficace o inefficace. Nel primo caso, il transfer è definito transfer positivo, nel secondo caso transfer negativo ${ }^{3}$. Ad esempio, nell'interlingua di spagnolo L2 un transfer positivo è la produzione della preposizione italiana "con", coincidente sia formalmente sia funzionalmente con la preposizione spagnola con; invece un caso di transfer negativo è rappresentato dalla produzione della preposizione italiana "per", che non ha corrispondenti formali in spagnolo bensì i due corrispondenti funzionali por e para, fra cui il parlante deve scegliere per veicolare uno dei significati espressi dalla preposizione italiana.

L'intercomprensione può trarre beneficio non solo dal transfer positivo, ma anche dal transfer negativo, dal momento che questo fa notare al parlante le differenze fra la lingua target e le altre conosciute, e contribuisce alla ricostruzione della

Il transfer negativo è più evidente, poiché dà luogo ad errori e possibili incomprensioni fra i parlanti, tanto da essere definito anche come transfer scoperto, a differenza del transfer positivo, definito anche transfer coperto, in quanto indistinguibile dagli elementi prodotti in modo target perché acquisiti dall'apprendente (Andorno et al. 2017). 
grammatica e del lessico della lingua target. L'esito inefficace del transfer negativo, determinando un punto di incomprensione fra gli interlocutori, apre una sequenza di negoziazione del significato che porta il parlante a notare l'inadeguatezza della sua scelta linguistica e a comprendere nell'interazione con il parlante più competente quale sia l'elemento della lingua target da usare al posto dell'elemento erroneamente trasferito dall'altra lingua. Questo è particolarmente rilevante nel caso dei cosiddetti «falsi amici» (Koessler / Derocquigny 1928), ovvero parole di due lingue che condividono la forma ma non il significato, come l'aggettivo spagnolo embarazada simile formalmente all'aggettivo italiano 'imbarazzata' ma di significato ben diverso, in quanto corrispondente semanticamente all'aggettivo italiano 'incinta'.

L'esempio del transfer sp. embarazada - it. "imbarazzata" mostra, in aggiunta, come il transfer possa essere innescato non solo da trasparenza totale, quanto anche da una trasparenza parziale fra due elementi non totalmente sovrapponibili in due lingue. Un altro caso di trasparenza parziale, questa volta a livello morfologico invece che lessicale, è costituito dai morfemi dell'imperfetto (sp. -ba-it. "-va"). Nelle ricerche sull'intercomprensione si documentano frequenti casi di transfer attivati oltre che da trasparenza totale o parziale anche dalla cosiddetta trasparenza diagonale (Degache / Masperi 1998; Vilaginés Serra 2007; García Castanyer / Vilaginés Serra 2010), ovvero la somiglianza fra due parole non corrispondenti bensì appartenenti allo stesso campo semantico nelle due lingue, come il termine francese enfant, 'bambino', comprensibile per un parlante che conosce l'italiano sulla base non del traducente diretto 'bambino', ma di parole come "infanzia" e "infantile", in cui si ritrova lo stesso morfema lessicale della parola francese enfant. Inoltre, il transfer può avvenire fra lingue non affini fra loro, come nella comprensione da parte di un romanofono del termine francese danger 'pericolo', che non coincide, neppure parzialmente o diagonalmente, con termini di altre lingue romanze, bensì con l'inglese danger, 'pericolo'.

Il parlante è dunque spinto a riflettere sulle lingue nel loro complesso: invece di fermarsi ad una comparazione superficiale fra singoli elementi è stimolato a ricercare connessioni fra l'intero lessico di tutte le risorse linguistiche del suo repertorio. Quanto più il parlante è plurilingue, tanto più agevole sarà il confronto fra diversi lessici e grammatiche per individuare somiglianze ed accedere al significato degli elementi nella nuova lingua. Oltre al ricorso a tutte le risorse linguistiche, dal momento che il confronto fra due sole lingue può non essere sufficiente, come testimonia il caso fr. danger $>$ ingl danger $>$ it. 'pericolo', un compito di intercomprensione mette in campo le competenze metalinguistiche del parlante, dato che non può fermarsi all'osservazione delle parole ma deve entrare nei meccanismi di formazione degli elementi linguistici, come nel caso della trasparenza diagonale esemplificato da fr. enfant > it. "infanzia", "infantile" > it. "bambino".

\subsection{L'intercomprensione fra spagnolo e italiano}

Nell'ambito delle lingue romanze, particolarmente stretta è l'affinità fra italiano e spagnolo, le due lingue oggetto di studio nella presente ricerca. Tale prossimità strutturale si riflette su tutti i piani di analisi linguistica e facilita tanto l'intercomprensione fra le due lingue (Compagno / Di Gesù 2007; Cortés Velásquez 2016; Tombolini 2018), quanto l'acquisizione sia dello spagnolo da parte di italofoni (Calvi 2001, 
2004a, 2004b) sia dell'italiano da parte di ispanofoni (Schmid 1994a, 1994b; Rosi 2009, 2020a).

Nel percorso acquisizionale la madrelingua affine alla lingua target ha un ruolo importante, soprattutto nelle prime fasi (Calvi 1995; Bailini 2016; Della Putta 2017; Cappelli 2019): gli apprendenti saltano le fasi prebasiche e basiche di interlingua e partono da uno stadio acquisizionale più avanzato rispetto agli apprendenti la cui L1 non è affine alla lingua target, in quanto consapevoli di potersi affidare alle regole e al lessico della madrelingua per comunicare, almeno per raggiungere gli scopi comunicativi essenziali. Ad esempio, la comparazione fra apprendenti ispanofoni e tedescofoni di italiano L2 nell'uso delle forme del passato mostra un notevole vantaggio iniziale degli apprendenti con L1 spagnola nella produzione dell'imperfetto, in quanto tale tempo verbale ha un corrispondente diretto nel sistema verbale spagnolo mentre è assente nel sistema verbale tedesco (Rosi 2009). Se gli apprendenti tedescofoni incontrano problemi nell'acquisizione della funzione dell'imperfetto e lo producono poco frequentemente e in modo spesso non target, per gli apprendenti ispanofoni il suo uso è precoce e target fin dalle prime occorrenze nell'interlingua, in quanto trasferito dalla L1. Tale vantaggio iniziale si riduce poi nel corso dell' acquisizione via via che aumenta l'esposizione all'input della lingua target e la sua analisi da parte dell'apprendente ${ }^{4}$.

L'iniziale facilità nell'interagire con i parlanti della lingua target sulla base della L1 può comportare come rovescio della medaglia la fossilizzazione di strutture della $\mathrm{L} 1$ divergenti dalla $\mathrm{L} 2$ finanche negli stadi avanzati di interlingua. La fossilizzazione può riguardare strutture di qualsiasi livello di analisi linguistica, come esemplificato dalle seguenti produzioni scritte di apprendenti ispanofoni di italiano LS nel contesto guidato dell'università di Granada: prodocto, megliore, maraviglioso, il specchio, sopra tutto, hygiene, prática, ho andato, uni consigli, Marco e Lidia sono venuti con i suoi figli (De Benedetti 2006). La fossilizzazione si verifica quando l'apprendente presuppone analogie fra la L1 e la L2 anche per gli elementi che non sono corrispondenti e non presta l'attenzione necessaria a notare le divergenze fra madrelingua e lingua target e ad acquisire le relative strutture della L2.

Nell'italiano degli immigrati ispanofoni in Svizzera, Schmid (1994a) rileva infatti una tendenza al minimo sforzo cognitivo, che si basa sulla prima delle tre strategie acquisizionali nell'apprendimento di lingue affini definite dall'autore. Queste sono (Schmid 1994a, 1994b):

a. la strategia della coerenza, che consiste nel riconoscimento delle analogie fra L1 e L2 e nella produzione delle forme e delle regole coerenti fra le due lingue, generando talvolta transfer negativi come la sovraestensione di alcune forme L2 sulla base del modello della L1, quale la combinazione fra la perifrasi progressiva e le forme verbali perfettive in italiano secondo lo schema spagnolo: interlingua e mentre è stato prendendo il pane $<$ spagnolo y mientras estuvo tomando el pan (Rosi 2009: 114);

b. la strategia della corrispondenza, che riguarda l'individuazione delle corrispondenze fra forme e funzioni diverse fra L1 e L2 e la conversione degli schemi della L1 secondo il modello della L2, portando a possibili transfer

Tagliavini (1947) afferma che lo spagnolo è una delle lingue più difficili da apprendere per un italofono, che può raggiungere facilmente un livello di competenza intermedio ma farà particolare fatica a superare tale livello e a padroneggiare la lingua in modo accurato. 
negativi anche in questo caso, ad esempio la conversione del morfema del participio passato spagnolo - do nell'italiano "-to" e la sua sovraestensione a verbi dal passato non regolare come corruto invece di "corso" sul modello dello spagnolo corrido;

c. la strategia della divergenza, costituita dalla scoperta di strutture differenti fra L1 e L2 di cui non si è in grado di ricostruire il modello in L2, che disorienta l'apprendente e può causare produzioni non target distanti sia dalla L1 sia dalla L2 e casi di ipercorrettismo, il fenomeno per cui gli apprendenti si correggono in maniera eccessiva, ad esempio evitando strutture corrispondenti alla L1 per non commettere errori dopo aver sperimentato la non totale congruenza fra L1 e L2, come l'uso frenato della perifrasi progressiva in apprendenti ispanofoni di italiano LS presso l'università di Siviglia (Rosi 2020).

La strategia della congruenza è molto adottata dagli apprendenti la cui L1 è affine alla L2, come il caso dell'italiano e dello spagnolo (Ferrario 2009): ciò comporta sia il ricorso alle forme più semplici e frequenti per non cimentarsi in strutture complesse che non si dominano a fondo, fermandosi ad una competenza linguistica ridotta, rilevata già da Dabène (1975) in apprendenti francofoni dello spagnolo, sia fenomeni di fossilizzazione. Gli studi sull'acquisizione dell'italiano di ispanofoni e dello spagnolo di italofoni di Della Putta $(2016,2017)$ mostrano come l'insegnamento riduca i casi di fossilizzazione dell'accusativo preposizionale (Juan llama a Maria, 'Giovanni chiama $\varnothing$ Maria') e dell'articolo pre-possessivo ( $\varnothing$ mi coche es rojo, 'la mia auto è rossa') grazie ad interventi didattici espliciti, mirati all'analisi dell'input L2 con diverse tecniche di focus on form e di textual enhancement, manipolazione grafica dello stimolo per guidare l'attenzione degli studenti a notare specifici elementi.

L'affinità fra l'italiano e lo spagnolo ha un effetto facilitatorio maggiore sulle competenze di comprensione che di produzione (Calvi 2004a). Nelle attività di comprensione di testi in una lingua affine alla L1, la strategia della coerenza è infatti fortemente attivata dalla frequente trasparenza degli elementi linguistici (Calvi 2004a, 2004b; Cortés Velásquez 2016): la somiglianza fra le strutture delle due lingue rende le parole e le regole della lingua target da subito riconoscibili per il parlante di una lingua affine. Dal momento che l'associazione fra le parole e i significati della L2 procede attraverso il filtro degli schemi parola / significato della L1 (Bettoni 2001), quanto più la L1 e la L2 sono simili, tanto più tale associazione è facile e rapida. Grazie alla trasparenza dunque, un parlante ispanofono e un italofono sono in grado di intercomprendersi con relativa facilità e accuratezza, pur non avendo mai studiato l'uno la lingua dell'altro. La trasparenza degli elementi lessicali e grammaticali ${ }^{6}$ infatti permette l'accesso al significato complessivo del testo, che costituisce la base per comprendere la nuova lingua e per ricostruire il significato degli elementi non

Lo studio di Rosi (2020) evidenzia non tanto la mancata accuratezza nella produzione della perifrasi progressiva nell'italiano di ispanofoni quanto la difficoltà nel padroneggiarne l'uso, dal momento che gli apprendenti evitano tale struttura in contesti in cui gli italofoni nativi la producono. Questo comportamento linguistico può dipendere dal fatto che gli apprendenti hanno notato nell'input di italiano la minor frequenza della perifrasi progressiva rispetto allo spagnolo, sperimentandone l'assenza in contesti in cui si sarebbero aspettati l'uso della perifrasi da parte degli italofoni nativi, e ciò li porta ad evitarla in contesti in cui la userebbero, per allinearsi il più possibile al modello che si sono costruiti della L2 ed essere sicuri di non commettere errori.

6 Per quanto riguarda la morfologia, la trasparenza fra italiano e spagnolo agevola il riconoscimento delle regole di formazione delle parole, con l'esclusione della morfologia derivativa in cui la diversa distribuzione di morfemi omofoni crea confusione negli apprendenti (Calvi 1999). 
compresi attraverso l'analisi del contesto e la comprensione globale del messaggio. Dall'altra parte, la trasparenza fra lingue affini è responsabile del fraintendimento delle parole che l'apprendente pensa di conoscere («words you think you know», Laufer 1997), come le parole simili nella forma ma non nel significato, il fenomeno dei falsi amici discusso nel par. 1.1., o le parole polisemiche che condividono in due lingue solo alcune accezioni semantiche, ad esempio la parola spagnola pantano, che indica lo stesso referente della parola italiano "pantano", ma anche il significato di lago artificiale, non atteso e pertanto non compreso da studenti italofoni di spagnolo, portati ad associare alla parola spagnola unicamente il significato a loro noto in quanto corrispondente alla L1, nonostante gli indizi contestuali (Calvi 2001).

Un ulteriore vantaggio dell'affinità fra la L1 e la L2 nella comprensione del testo riguarda i processi adottati per accedere al significato (Calvi 2004b). Gli apprendenti con L1 affine alla L2 applicano principalmente processi di livello superiore (topdown), orientati sui concetti e basati sul contesto, come fanno i parlanti nativi; gli apprendenti con L1 non affine alla L2 costruiscono il significato del testo con procedimenti di livello inferiore (bottom-up), partendo dalle singole forme linguistiche che vengono poi integrate nella rappresentazione del significato complessivo, dal momento che le opacità lessicali e grammaticali sono troppo numerose per permettere l'attivazione dei processi di comprensione usati per la L1. I processi top-down permettono agli apprendenti di ricostruire il significato anche di termini opachi basandosi sul contesto fin dalle prime fasi acquisizionali, come messo in luce da ricerche sulla comprensione di testi accademici italiani da studenti ispanofoni iscritti a programmi di scambio internazionale e da nativi (Rosi 2020b). Se nei parlanti nativi il ricorso al contesto svolge un ruolo facilitatorio nella comprensione del testo, per i non nativi sono documentati, però, anche casi di fraintendimento del significato di singole parole sulla base di indizi contestuali, come ad esempio quelli emersi nei dati qui analizzati (cfr. par. 3.2.1). Inoltre, i processi top-down possono indurre all'evitamento di parole opache o ambigue. Calvi (2001) riporta come studenti universitari di spagnolo di livello intermedio scelgano talvolta di evitare di tradurre in italiano la parola nieve ('neve'), trasparente e pienamente comprensibile, perché nei loro schemi culturali la neve non è associata al clima della Spagna, concepita in maniera stereotipata come un paese caldo e soleggiato durante tutto l'anno. Tali studenti saltano nella traduzione in italiano i punti del testo in cui occorre la parola nieve, facendo riferimento alla rappresentazione concettuale che hanno in mente piuttosto che alla trasparenza della parola rispetto alla L1. Questo è un esempio di come nella fase intermedia dell'apprendimento a U, per ipercorrettismo, l'apprendente possa tendere a evitare di trasferire nella L2 le strutture della L1, dopo aver sperimentato che non tutti i transfer raggiungono l'efficacia comunicativa, secondo la strategia della divergenza, e a distaccarsi il più possibile dalla L1 per evitare errori.

\section{3. Le applicazioni didattiche dell'intercomprensione}

Gli studi sull'intercomprensione (Blanche-Benveniste / Valli 1997; Benucci 2005, 2015; De Carlo 2011; Bonvino / Jamet 2016) sono nati nel quadro glottodidattico dell'approccio plurale all'apprendimento/ insegnamento delle lingue ${ }^{7}$, mirato non

Il documento CARAP - Un Cadre de Référence pour les Approches Plurielles des langues et des cultures. Compétences et ressources (Candelier et al. 2007), che costituisce un'ideale continuazione del Quadro Comune Euro- 
tanto all'acquisizione di una singola lingua, di cui tramite l'intercomprensione si possono sviluppare solo abilità ricettive e parziali, quanto al potenziamento di competenze trasversali di riflessione metalinguistica e di comprensione, che i parlanti possono raffinare con attività su lingue fra loro affini e poi applicare a lingue fra loro diverse. Nei percorsi didattici basati sull'intercomprensione sono proposte attività di lettura, ascolto e comprensione di più lingue nello stesso momento, partendo da lingue strettamente imparentate per sfruttare la somiglianza strutturale fra esse e facilitare il confronto interlinguistico da parte del parlante. L'attività traduttiva esercitata nell'intercomprensione ha un effetto positivo sulla competenza metalinguistica, potenziandola in maniera trasversale per tutte le lingue del repertorio, sia sulle L2, sia sulla L1, di cui è stimolata un'analisi attenta e approfondita in funzione della comprensione della nuova lingua che il parlante ha di fronte. Inoltre, le strategie trasversali di comprensione che vengono ad essere consolidate nell'intercomprensione, come la segmentazione del testo in unità tematiche e l'individuazione delle informazioni più rilevanti per la comprensione globale (Bonvino 2012), contribuiscono a migliorare il metodo di studio delle lingue e, più in generale, ad imparare ad apprendere e formare nuova conoscenza.

Numerosi progetti di ricerca si sono incentrati sull'intercomprensione fra lingue romanze, come quelli del gruppo di ricerca dell'università Stendhal di Grenoble, coordinato da Louise Dabène e in seguito da Christian Degache (GALATEA, GALANET, GALAPRO; Dabène / Degache 1996), e l'équipe dell'Université Aix-Marseille, coordinata da Claire Blanche-Benveniste (Eurom4, Eurom5, EuroComRom, cf. Blanche-Benveniste et al. 1997; Bonvino et al. 2011). Quest'ultimo progetto (EuroCom, http://www.eurocomprehension.eu/), rivolto originariamente ad un pubblico di studenti universitari di L1 tedesca con conoscenze di francese, e poi ampliatosi ${ }^{8}$, ha portato all'elaborazione della tecnica die sieben Siebe (Klein / Stegmann 1999), dei 'sette setacci', che, con la metafora dell'atto di setacciare ciclicamente il testo, offre indicazioni su come ricercare indizi utili a ricostruire analogie interlinguistiche per accedere alla comprensione.

I sette domini proposti per attivare il confronto interlinguistico e il riconoscimento delle strutture simili a quelle note sono: il lessico internazionale, composto da termini, frequentemente di origine greca o latina, condivisi da un largo campione di lingue, non solo romanze, ricorrenti nei testi di argomento tecnico-scientifico, politico, sportivo; il lessico panromanzo, costituito da parole del lessico di base, condivise fra le lingue romanze in quanto derivate dal latino; le corrispondenze fonologiche, ovvero l'associazione fra fonemi e grafemi, che aiutano a ricostruire le evoluzioni diacroniche dal latino alle diverse lingue romanze; le corrispondenze fra grafia e pronuncia, per comprendere le convenzioni ortografiche di ciascuna lingua; l'ordine sintattico, comune nelle lingue romanze, che facilita la scoperta delle funzioni sintattiche degli elementi linguistici sulla base della loro posizione all'interno della frase; la morfosintassi, che agevola la ricostruzione della struttura interna delle parole; gli affissi, condivisi fra le lingue romanze in quanto di origine greca o latina,

peo di Riferimento per la conoscenza delle lingue (Consiglio d'Europa 2001), individua quattro principali approcci plurali: la sensibilizzazione alle lingue o éveil aux langues, l'approccio interculturale, l'approccio integrato alle lingue e l'intercomprensione ((http://carap.ecml.at/Handbook/tabid/2602/language/fr-FR/Default.aspx).

8 Per indagare l'intercomprensione in altri raggruppamenti di lingue strettamente imparentate fra loro, il Progetto ha dato vita anche alle sezioni EuroComGerm, su lingue germaniche (danese, inglese, nederlandese, svedese, tedesco), e EuroComSlav, su lingue slave (bielorusso, bulgaro, ceco, croato, polacco, ucraino). 
che permettono di comprendere una parte del significato delle parole derivate e di segmentarle in diverse componenti.

Com'è evidente, la tecnica dei "sette setacci" individua piste di analisi e confronto applicabili anche a lingue non imparentate fra loro. In questa prospettiva rientrano il Progetto $E u \& I^{9}$, che propone attività su lingue europee non affini fra loro mirando alla sensibilizzazione dei cittadini dell'Unione Europea verso le verso il plurilinguismo, e i progetti InterCom e Intermar ${ }^{10}$, che sperimentano l'intercomprensione fra lingue anche molto distanti fra loro e documentano l'impiego di strategie di comprensione trasversali, come il ricorso alle conoscenze enciclopediche, l'attivazione di inferenze a partire dal contesto linguistico o paralinguistico, il riconoscimento del lessico internazionale e di regole morfologiche e sintattiche condivise da più lingue.

Comune a tutti i progetti sull'intercomprensione è l'approccio induttivo centrato sul parlante, che si dimostra altamente motivante ed efficace. Le attività di intercomprensione si articolano nella lettura di un testo, accompagnata sempre più frequentemente dall'ascolto del testo letto da un parlante nativo, e nella trasposizione orale del testo in L1, co-costruita fra i partecipanti attraverso i contributi dei singoli e la condivisione dei punti più difficili del testo e delle soluzioni adottate. La trasposizione in L1 risulta dunque lontana dalle tecniche dell'approccio comunicativo-grammaticale, fondato sulla memorizzazione di regole grammaticali e sulla loro applicazione a compiti traduttivi allo scopo di migliorarne l'accuratezza; bensì è orientata all'elicitazione delle strategie di comprensione dei partecipanti con la tecnica «Think Aloud Protocol» (van Someren et al. 1994), composta da un esercizio linguistico e dalla successiva verbalizzazione dei processi cognitivi compiuti per eseguirlo. Il parlante è coinvolto in un'attività di problem solving da svolgere in autonomia, ricorrendo alle conoscenze linguistiche e alla competenza metalinguistica in suo possesso. È un compito comunicativo attivo, che fa acquisire la consapevolezza che le conoscenze linguistiche già acquisite possono aprire l'accesso a lingue mai studiate prima ed aumenta tanto la fiducia nelle capacità personali quanto la motivazione allo studio di nuove lingue.

Un altro aspetto importante è che nelle attività di intercomprensione i parlanti non hanno l'ansia di commettere errori in quanto non sentono di dover dimostrare di conoscere la lingua, non avendola mai studiata (Bonvino 2012), e si sorprendono della propria capacità di comprendere nuove lingue (Bonvino / Cortés Velásquez 2016b). Le aspettative del parlante sulla possibilità di risolvere il compito sono nulle, per cui ogni scelta efficace è percepita come un successo. Dall'altro lato, i parlanti si accorgono delle diversità fra le lingue, anche fra quelle affini, e vengono spronati a

Il Progetto EU\&I - European Awareness and Intercomprehension (http://www.eu-intercomprehension.eu/) propone attività di intercomprensione in contesti comunicativi legati ad una specifica ambientazione, una camera di albergo, che hanno come stimolo una guida turistica, previsioni meteorologiche o istruzioni per accendere il televisore, nell'ottica di presentare casi concreti di intercomprensione nella vita quotidiana e stimolare i partecipanti a trovare strategie di comunicazione plurilingue utili per dialogare con chi entra per la prima volta nei loro paesi senza conoscerne la lingua. Nel progetto rientrano 11 lingue (bulgaro, francese, greco, inglese, italiano, nederlandese, portoghese, spagnolo, svedese, tedesco, turco), connesse fra loro da differenti legami genealogici, come le lingue romanze, germaniche, slave e lingue indoeuropee isolate, quale il greco, insieme ad una lingua non imparentata con nessuna delle precedenti e appartenente alla famiglia altaica (turco).

10 Il Progetto InterCom (http://www.intercomprehension.eu/) propone attività di intercomprensione di greco, portoghese e tedesco nell'ambito delle situazioni comunicative del turismo, come la prenotazione di un alloggio, la ricerca di un indirizzo stradale o la ricetta di un piatto tipico. Il Progetto Intermar (http://www.intermar.ax) è rivolto all'intercomprensione in comunicazioni di argomento marittimo e navale in lingue baltiche, germaniche, romanze e in russo. 
non fermarsi ad un primo contatto superficiale con la lingua target, ma ad approfondirne la conoscenza. Questa spinta motivazionale ha un ruolo chiave nella promozione dell'apprendimento di lingue affini alla L1, che predispone alla comprensione di ulteriori lingue (Balboni 2018), e, più in generale, nella promozione del plurilinguismo, inteso come conoscenza di più varietà e lingue che non si sviluppa in maniera lineare e cumulativa lingua per lingua, ma in forma integrata e trasversale fra tutte le lingue del repertorio di un parlante (Consiglio d'Europa 2001).

\section{Un esperimento di intercomprensione}

\subsection{Partecipanti}

I partecipanti all'esperimento di intercomprensione sono 20 studenti dell'ultimo anno del corso di studi magistrali dell'Università di Salerno, privi di alcuna conoscenza pregressa di lingua spagnola: una metà (gruppo A) è iscritta al corso di studi in Lingue e Letterature Moderne e studia lingue non romanze (inglese, tedesco o russo); l'altra metà (gruppo B) è iscritta a corsi di studi in ingegneria (8 studenti su 10), scienze motorie o scienze dello spettacolo e della produzione multimediale. Entrambi i gruppi hanno pari livello di istruzione e sono dotati di capacità critiche di osservazione, analisi, riflessione e astrazione.

Dalle risposte fornite in un iniziale questionario scritto, mirato a delineare il profilo linguistico degli studenti, i due gruppi risultano comparabili per età, area di provenienza (Campania) e L1: tutti i partecipanti sono italofoni nativi, con un'alta percentuale di studenti che conoscono ed usano il dialetto locale. Differenze emergono, invece, per quanto riguarda il repertorio linguistico individuale e l'atteggiamento verso l'apprendimento delle lingue (Tabelle 1 e 2).

\begin{tabular}{|l|l|l|l|l|}
\hline Studente & \multicolumn{1}{|c|}{ L1 } & $\begin{array}{c}\text { Lingue studiate } \\
\text { all'università } \\
\text { (livello QCER) }\end{array}$ & $\begin{array}{l}\text { Altre lingue conosciute } \\
\text { (livello QCER) }\end{array}$ & $\begin{array}{l}\text { Ti piace im- } \\
\text { parare nuove } \\
\text { lingue? }\end{array}$ \\
\hline S1 & $\begin{array}{l}\text { Italiano e } \\
\text { dialetto }\end{array}$ & $\begin{array}{l}\text { Inglese (C2) } \\
\text { Tedesco (C2) }\end{array}$ & $\begin{array}{l}\text { Francese (B1/B2) } \\
\text { Latino }\end{array}$ & Molto \\
\hline S2 & $\begin{array}{l}\text { Italiano e } \\
\text { dialetto }\end{array}$ & $\begin{array}{l}\text { Inglese (C2) } \\
\text { Russo (C2) }\end{array}$ & $\begin{array}{l}\text { Francese (A1) Ebraico } \\
\text { A1) } \\
\text { Latino }\end{array}$ & Molto \\
\hline S3 & Italiano & $\begin{array}{l}\text { Inglese (C1) } \\
\text { Russo (B2/C1) }\end{array}$ & $\begin{array}{l}\text { Francese (A2) Estone } \\
\text { (A1) } \\
\text { Latino }\end{array}$ & Molto \\
\hline S5 & $\begin{array}{l}\text { Italiano e } \\
\text { dialetto }\end{array}$ & $\begin{array}{l}\text { Inglese (B2/C1) } \\
\text { Russo (B1+) }\end{array}$ & Latino & Molto \\
\hline Iialiano e & $\begin{array}{l}\text { Inglese (B2/C1) } \\
\text { Russo (B2) }\end{array}$ & $\begin{array}{l}\text { Francese (B1) Tedesco } \\
\text { (B1) } \\
\text { Latino }\end{array}$ & Molto \\
\hline
\end{tabular}




\begin{tabular}{|l|l|l|l|l|}
\hline S7 & Italiano & $\begin{array}{l}\text { Inglese (C1+) } \\
\text { Russo (B1+) }\end{array}$ & $\begin{array}{l}\text { Francese (A2) Tedesco } \\
(\mathrm{A} 2) \\
\text { Latino }\end{array}$ & Abbastanza \\
\hline S8 & $\begin{array}{l}\text { Italiano e } \\
\text { dialetto }\end{array}$ & $\begin{array}{l}\text { Inglese (B1) Russo } \\
(\mathrm{A} 2+)\end{array}$ & $\begin{array}{l}\text { Tedesco (A2+) Portoghe- } \\
\text { se (A1) } \\
\text { Latino }\end{array}$ & Molto \\
\hline S9 & $\begin{array}{l}\text { Italiano e } \\
\text { dialetto }\end{array}$ & $\begin{array}{l}\text { Inglese (C1) } \\
\text { Tedesco (C1) }\end{array}$ & $\begin{array}{l}\text { Francese (B2) } \\
\text { Latino }\end{array}$ & Molto \\
\hline S10 & $\begin{array}{l}\text { Italiano e } \\
\text { dialetto }\end{array}$ & $\begin{array}{l}\text { Inglese (C1) } \\
\text { Tedesco (B2) }\end{array}$ & $\begin{array}{l}\text { Francese (B1) } \\
\text { Latino }\end{array}$ \\
\hline
\end{tabular}

Tabella 1. Il gruppo A: gli studenti di lingue

\begin{tabular}{|c|c|c|c|c|}
\hline Studente & $\begin{array}{l}\text { Corso di } \\
\text { studi }\end{array}$ & L1 & $\begin{array}{l}\text { Altre lingue conosciute } \\
\quad \text { (livello QCER) }\end{array}$ & $\begin{array}{l}\text { Ti piace imparare } \\
\text { nuove lingue? }\end{array}$ \\
\hline S11 & $\begin{array}{l}\text { Ingegneria } \\
\text { informatica }\end{array}$ & $\begin{array}{l}\text { Italiano e } \\
\text { dialetto }\end{array}$ & $\begin{array}{l}\text { Inglese (A2) } \\
\text { Tedesco (A1) }\end{array}$ & Abbastanza \\
\hline $\mathrm{S} 12$ & $\begin{array}{l}\text { Ingegneria } \\
\text { meccanica }\end{array}$ & $\begin{array}{l}\text { Italiano e } \\
\text { dialetto }\end{array}$ & $\begin{array}{l}\text { Inglese }(\mathrm{B} 1+) \\
\text { Latino }\end{array}$ & Abbastanza \\
\hline S13 & $\begin{array}{l}\text { Ingegneria } \\
\text { informatica }\end{array}$ & $\begin{array}{l}\text { Italiano e } \\
\text { dialetto }\end{array}$ & $\begin{array}{l}\text { Inglese (B1) } \\
\text { Tedesco (A1) }\end{array}$ & Abbastanza \\
\hline S14 & $\begin{array}{l}\text { Ingegneria } \\
\text { chímica }\end{array}$ & $\begin{array}{l}\text { Italiano e } \\
\text { dialetto }\end{array}$ & $\begin{array}{l}\text { Inglese (B1) } \\
\text { Francese (A2) } \\
\text { Latino }\end{array}$ & Abbastanza \\
\hline $\mathrm{S} 15$ & $\begin{array}{l}\text { Ingegneria } \\
\text { informatica }\end{array}$ & $\begin{array}{l}\text { Italiano e } \\
\text { dialetto }\end{array}$ & $\begin{array}{l}\text { Inglese (B1) } \\
\text { Tedesco (A1) }\end{array}$ & Abbastanza \\
\hline S16 & $\begin{array}{l}\text { Ingegneria } \\
\text { informatica }\end{array}$ & Italiano & Inglese (B1) & No \\
\hline S17 & $\begin{array}{l}\text { Ingegneria } \\
\text { civile }\end{array}$ & $\begin{array}{l}\text { Italiano e } \\
\text { dialetto }\end{array}$ & $\begin{array}{l}\text { Inglese (B2) } \\
\text { Tedesco (A1) }\end{array}$ & Molto \\
\hline S18 & $\begin{array}{l}\text { Ingegneria } \\
\text { informatica }\end{array}$ & $\begin{array}{l}\text { Italiano e } \\
\text { dialetto }\end{array}$ & $\begin{array}{l}\text { Inglese (A2) } \\
\text { Tedesco (A1) }\end{array}$ & Abbastanza \\
\hline S19 & $\begin{array}{l}\text { Scienze } \\
\text { dello spetta- } \\
\text { colo e della } \\
\text { produzione } \\
\text { multimediale }\end{array}$ & $\begin{array}{l}\text { Italiano e } \\
\text { dialetto }\end{array}$ & $\begin{array}{l}\text { Inglese (B2) } \\
\text { Francese (A2) } \\
\text { Latino }\end{array}$ & Molto \\
\hline S20 & $\begin{array}{l}\text { Scienze } \\
\text { motorie }\end{array}$ & $\begin{array}{l}\text { Italiano e } \\
\text { dialetto }\end{array}$ & $\begin{array}{l}\text { Inglese (A2) } \\
\text { Francese (A1) } \\
\text { Latino }\end{array}$ & Abbastanza \\
\hline
\end{tabular}

Tabella 2. Il Gruppo B: gli studenti di discipline non linguistiche

Il repertorio linguistico dei partecipanti è plurilingue in entrambi i gruppi, ma gli studenti di lingue dichiarano di conoscere più lingue (alcuni studenti del gruppo $\mathrm{A}$ conoscono fino a 6 o 7 lingue) e a livelli di competenza più avanzati: fra il $\mathrm{B} 2$ e il C2 nel gruppo A, dall'A1 al B1, con pochi casi di B2, nel gruppo B. Inoltre, nel gruppo 
A è molto più diffuso lo studio nella scuola secondaria superiore del latino (100\% vs. $40 \%$ ) e del francese ( $80 \%$ vs. $30 \%$ ). È da sottolineare che entrambi i gruppi sono composti da parlanti che hanno studiato lingue classiche e moderne a scuola e dispongono di un repertorio plurilingue, ma nei cinque anni precedenti alla raccolta dati l'esposizione alle lingue straniere si è molto diversificata fra gli iscritti al corso di lingue, abituati ad attività linguistiche e traduttive quotidiane nell'ambito di un'istruzione formale che prevede spiegazioni grammaticali, riflessioni metalinguistiche e confronti interlinguistici da parte di docenti esperti e dei pari, e gli iscritti agli altri corsi, concentrati su materie ingegneristiche e materie umanistiche non legate alla lingua, come la pedagogia, l'antropologia, la storia dell'arte, i quali ricorrono alle lingue straniere in poche occasioni di vacanza o di comunicazioni sui social networks. La diversità quantitativa e qualitativa del contatto con le lingue straniere durante i cinque anni del percorso universitario ha portato i due gruppi ad un differente grado di maturazione non solo delle competenze linguistico-comunicative, ma anche della competenza metalinguistica, che è stata stimolata, allenata e perfezionata nel gruppo A in misura molto maggiore rispetto al gruppo B.

Per quanto riguarda l'atteggiamento verso l'apprendimento delle lingue, gli studenti del gruppo A si dichiarano tutti molto interessati ad imparare nuove lingue, mentre nel gruppo B l'interesse ad apprendere nuove lingue è inferiore, come prevedibile dato il percorso universitario scelto, per quanto non negativo, con l'eccezione di un unico partecipante che dichiara di non avere nessun interesse a conoscere nuove lingue.

\subsection{Metodologia}

L'esperimento è stato condotto da una ricercatrice di età pari ai partecipanti, con una buona conoscenza teorica dell'intercomprensione ${ }^{11}$. Tutti gli studenti hanno accettato di partecipare all'esperimento con interesse e curiosità. Nessuno di loro conosceva il termine intercomprensione, che è stato spiegato dalla ricercatrice per introdurre l'attività, né tecniche intercomprensive. Come suggerito da Jamet (2010), la lingua del questionario e dell'interazione è stata la L1 dei partecipanti, per evitare difficoltà nella comprensione delle consegne e nella produzione delle risposte.

Lo stimolo è costituito da un testo scritto autentico: un articolo di cronaca sul terremoto verificatosi in Messico il 24 settembre 2017, pubblicato sul sito online del quotidiano El País (cfr. Appendice). Il testo è stato presentato senza un glossario o altra forma di aiuto linguistico e senza immagini, così da proporre agli studenti un'attività di lettura non facilitata. L'articolo consta di 495 parole, appartenenti a diversi ranghi di frequenza del lessico spagnolo: da parole del lessico di base a termini tecnici della varietà accademico-scientifica della geologia, pertinenti all'argomento del terremoto trattato nell'articolo.

I due gruppi hanno partecipato alla stessa sessione sperimentale, dalla durata di un'ora e trenta minuti ${ }^{12}$. Dopo aver compilato il questionario con le informazioni sul

1 L'esperimento, la trascrizione e la tokenizzazione dei dati, ideati da chi scrive, sono stati condotti da Francesca Napolitano nell'ambito della sua tesi di laurea magistrale in Lingue e Letterature Moderne presso l'Università di Salerno (Napolitano 2018).

12 L'esperimento si è svolto l'8 novembre 2017 dalle 9 alle 10,30 in un'aula del Dipartimento di Studi Umanistici dell'Università di Salerno. 
proprio background linguistico (cfr. 3.1.), gli studenti hanno ricevuto il testo per leggerlo e poi ne hanno ascoltato la lettura ad alta voce audioregistrata da un'ispanofona nativa $^{13}$ con il testo davanti. In seguito, hanno risposto a quattro domande di comprensione: a) Di cosa parla l'articolo?; b) Cos'ha permesso agli abitanti di Città del Messico di abbandonare le proprie case?; c) Cosa spiega Pérez Campos?; d) Perché le due vittime di Città del Messico sono state stroncate da un infarto? Le domande misurano la capacità di comprensione globale (a), analitica (b e c) e inferenziale (d) ${ }^{14}$, in un crescendo di complessità cognitiva che ha richiesto ai partecipanti diversi livelli di elaborazione linguistica e di operazioni logiche (Lumbelli 2009): dalla comprensione più generale alla più dettagliata, all'integrazione delle informazioni del testo con le conoscenze enciclopediche del lettore. Gli studenti hanno poi scritto la trasposizione del testo spagnolo in italiano ed infine hanno risposto a domande sulle difficoltà incontrate, le strategie adottate nei compiti precedenti e l'utilità della lettura accompagnata dall'ascolto. Queste fasi si sono svolte con un tempo massimo a disposizione dei partecipanti: 10 minuti per le domande di comprensione, 30 minuti per la trasposizione, 10 minuti per le domande conclusive. Infine, si è svolta ed è stata audioregistrata la correzione collettiva della trasposizione in italiano, in cui i partecipanti sono stati invitati a leggere a turno brani della propria trasposizione, condividendola e collaborando fra pari all'individuazione della soluzione migliore per ogni punto del testo con la guida della ricercatrice, che ha rivestito un ruolo di facilitatrice, sottolineando punti rimasti insoluti nella discussione, errori non riconosciuti dai partecipanti e contributi particolarmente significativi, sia sulle scelte linguistiche, che sulle riflessioni metalinguistiche degli studenti. Nelle varie fasi dell'esperimento sono state così raccolte verbalizzazioni metalinguistiche e metacognitive relative alle strategie di comprensione, sia scritte sia orali, sia individuali sia co-costruite fra pari.

\section{Analisi}

\subsection{La comprensione del testo}

Nelle analisi sono stati assegnati valori numerici secondo scale di valutazione in cui sono stati distinti i casi di errore e di omissione, in quanto espressione di diversi approcci al compito: l'errore mostra che lo studente ha tentato di risolvere un problema linguistico, facendo ricorso alle sue risorse linguistiche e metalinguistiche; l'omissione di risposta indica la rinuncia a svolgere il compito e sottolinea l'incapacità di elaborare una strategia, per quanto sbagliata, per fornire una proposta di soluzione. Le risposte date dagli studenti alle quattro domande di comprensione del testo sono state valutate secondo la seguente scala:

13 La lettura accompagnata dall'ascolto è un'attività facilitatoria che aiuta a chiarire le regole di conversione fonema-grafema della lingua obiettivo, anche grazie all'individuazione di eventuali differenze con il sistema della L1. L'ascolto si è fornito per una seconda lettura, dopo la prima individuale, affinché la scoperta della pronuncia delle parole del testo fungesse da conferma del significato dei termini compresi e aiutasse la disambiguazione dei termini non compresi, su cui gli studenti avevano già riflettuto nella prima lettura del testo.

14 La domanda 4 richiede di elaborare un'inferenza poiché nel testo non viene esplicitata la causa dell'infarto che ha stroncato due vittime. Il lettore è chiamato ad integrare il testo con le proprie conoscenze enciclopediche per ricostruire le conseguenze tipiche di un terremoto associabili all'infarto, come il forte spavento e lo sconvolgimento emotivo che rappresentano la risposta corretta alla domanda. 
a. 1 per le risposte corrette, come la seguente risposta alla domanda "Cosa spiega Pérez Campos?"

(1) St4. Dice che ogni sisma è diverso dall'altro. (estratto dal testo: Cada sismo se comporta de forma diferente);

b. 0.50 per le risposte scorrette, come la seguente risposta alla domanda "Cosa spiega Pérez Campos?"

(2) St7. Ogni sisma può avere da una ad otto repliche. (estratto dal testo: Cada sismo se comporta de forma diferente. El número de réplicas varía de uno a otro);

c. 0 per l'omissione di risposta.

Come si può osservare nella tabella 3 , il valore medio della correttezza delle risposte nei due gruppi è identico per la domanda di comprensione globale e molto simile nelle risposte alle domande di comprensione analitica (differenza di 0.10 e 0.20 punti) e alla domanda che richiede un'inferenza (differenza di 0.05 punti).

\begin{tabular}{|l|c|c|}
\hline & Gruppo A & Gruppo B \\
\hline Comprensione globale & 1 & 1 \\
\hline Comprensione analitica & 1 & 0.90 \\
\hline Comprensione analitica & 0.90 & 0.70 \\
\hline Comprensione inferenziale & 0.90 & 0.85 \\
\hline
\end{tabular}

Tabella 3. Correttezza delle domande di comprensione del testo (media per gruppo)

Il primo risultato che emerge dai dati è che entrambi i gruppi sono riusciti a comprendere bene il contenuto del testo spagnolo, dal momento che la correttezza delle risposte è sempre vicina al valore massimo (1). Il secondo risultato è che tutte le domande mostrano la forte analogia fra gli studenti di lingue e quelli di discipline non linguistiche. Nella prima domanda, tutti gli studenti hanno individuato l'argomento principale del testo, ovvero il terremoto verificatosi in Messico nel settembre 2017; nella seconda, solo uno studente del gruppo B non ha compreso che gli abitanti hanno abbandonato le proprie case per l'allarme suonato prima dell'inizio del terremoto; nella quarta, 8 studenti su 10 in entrambi i gruppi hanno saputo elaborare l'inferenza che la morte di due vittime era stata causata dallo spavento per il terremoto. La terza domanda è quella che ha creato un po' più di difficoltà. Nella correzione collettiva è emerso che la causa di tale difficoltà è stata il fraintendimento del significato di due parole: alcuni studenti hanno trasposto la parola cada, 'ciascuno', con 'questo' e la parola otro, 'altro', con 'otto', fornendo la risposta scorretta riportata in (2) e travisando il contenuto di questo punto del testo. 


\subsection{La trasposizione del testo in $\mathrm{L} 1$}

\subsubsection{Accuratezza lessicale}

In accordo con precedenti lavori sull'intercomprensione (Cortés Velásquez 2016), per misurare 1'accuratezza lessicale delle trasposizioni in L1 degli studenti, sia le 20 trasposizioni in italiano sia il testo dello stimolo spagnolo sono stati tokenizzati ${ }^{15}$ a livello di parola. Nei testi degli studenti, l'accuratezza lessicale delle trasposizioni di ciascun token è stata valutata secondo la seguente scala:

a. 1 per le trasposizioni lessicalmente corrette, come seísmo trasposto come "sisma" nell'esempio seguente:

(3) St10. Un sisma di magnitudine 6,1 (< Un seísmo de magnitud 6,1);

b. 0.75 per le trasposizioni lessicalmente approssimate, che riportano un termine semanticamente affine al traducente esatto, come pedimos trasposto come "invitiamo" invece di "chiediamo" nell'esempio seguente:

(4) St9. Invitiamo tutta la popolazione a mantenere la calma $(<$ Pedimos a toda la población mantener la calma);

c. $0, .50$ per le trasposizioni influenzate dal transfer negativo innescato dall'esistenza di falsi amici, come temblor trasposto come "tremore" invece di "scossa" nell'esempio seguente:

(5) St11. Un nuovo tremore scuote il centro e il sud del Messico (< Un nuevo temblor sacude el centro y sur de México);

d. 0.25 per le trasposizioni scorrette, come vez trasposto come "voce" al posto di "volta":

(6) St17. indicò a voce la Presidenza (< indicó a su vez la Presidencia);

e. 0 in caso di omissione, come l'omissione dell'avverbio alrededor 'circa', nell'esempio seguente:

(7) St1. ha causato fino ad ora 300 morti (< ha causado hasta el momento alrededor de 300 víctimas mortales).

Nella scala di accuratezza lessicale si sono distinti non solo gli errori dalle omissioni di risposta, ma anche tre diverse tipologie di errore, in base al processo di comprensione adottato per trasporre in L1: nella prima tipologia, lo studente comprende parzialmente il significato, individuando solo alcuni tratti semantici della parola spagnola, che traspone con un quasi-sinonimo del suo traducente esatto ("invitiamo"

\footnotetext{
La tokenizzazione è un'operazione di suddivisione del testo in unità minime di analisi, chiamate tokens.
} 
$<$ pedimos); nella seconda, lo studente non comprende nessun tratto semantico e cade nella trappola dei falsi amici ("tremore" < tremblor); nella terza, lo studente non comprende nessun tratto semantico della parola spagnola e la traspone con una parola italiana non collegata al traducente esatto, lasciandosi guidare da affinità morfofonologiche anche lievi o da errati indizi contestuali ("voce" < vez).

Le occorrenze di ciascuna tipologia di trasposizione nei due gruppi sono riportate nella Tabella 4.

\begin{tabular}{|l|c|c|}
\hline & Gruppo A & Gruppo B \\
\hline Trasposizioni corrette & 440.28 & 349.20 \\
\hline Trasposizioni approssimate & 4.57 & 2.70 \\
\hline Trasposizioni di falsi amici & 0.28 & 0.70 \\
\hline Trasposizioni scorrette & 6.41 & 6.10 \\
\hline Trasposizioni omesse & 43.43 & 139.70 \\
\hline
\end{tabular}

Tabella 4. Trasposizioni in L1 (media per gruppo)

In media, gli studenti di lingue mostrano un'accuratezza maggiore dei pari: le trasposizioni corrette superano di 91.08 punti quelle del gruppo B, le trasposizioni approssimate sono quasi il doppio, le trasposizioni influenzate dall'esistenza di falsi amici sono meno della metà e le omissioni sono un terzo. Tali dati sono riassunti nella Tabella 5, che mostra l'accuratezza media per gruppo, risultante dalla media delle somme dei valori delle diverse tipologie di trasposizione per ciascun testo prodotto dagli studenti.

\begin{tabular}{|l|c|c|}
\hline & Gruppo A & Gruppo B \\
\hline Accuratezza lessicale & 444.38 & 352.60 \\
\hline
\end{tabular}

Tabella 5. Accuratezza lessicale della trasposizione in L1 (media per gruppo)

Il gruppo $\mathrm{A}$ ha trasposto il testo in modo più accurato del gruppo $\mathrm{B}$, avvicinandosi maggiormente al valore massimo rappresentato dal numero totale dei token dello stimolo (495). Rispetto alla comprensione del contenuto (Tabella 3), la trasposizione del testo risulta più impegnativa per tutti gli studenti, come si evince dalla maggior distanza dei valori medi di entrambi i gruppi rispetto al valore massimo. Inoltre, il compito della trasposizione fa emergere un vantaggio del $18.5 \%$ per gli studenti di lingue rispetto a quelli di discipline non linguistiche. Una delle spiegazioni consiste nel fatto che quasi la metà del gruppo B (4 studenti su 10) non è riuscita a completare la trasposizione in L1 nel limite dato di 30 minuti, omettendo interi paragrafi della parte finale del testo ${ }^{16}$. Ciò indica che gli studenti di discipline non linguistiche hanno incontrato più difficoltà nella trasposizione in L1, impegnando più tempo nella scelta delle parole e mostrando il bisogno di rileggere più volte sia il testo stimolo, alla ricerca di indizi contestuali per ricostruire il significato di singole parole, sia la trasposizione fatta fino a quel punto, per trarre

16 La mancata trasposizione dell'ultima parte del testo input si riscontra in diversi esperimenti di intercomprensione (Cortés Velásquez 2016). 
supporto dalla coerenza logico-concettuale fra i vari blocchi tematici del testo per procedere nella trasposizione in L1.

Per quanto riguarda le difficoltà lessicali del testo, nonostante la superiorità nei tempi e nei risultati della trasposizione in L1 degli studenti di lingue, i due gruppi indicano come difficili le stesse parole e con la stessa frequenza (Tabella $6)$.

\begin{tabular}{|c|c|c|}
\hline & Gruppo A & Gruppo B \\
\hline $\begin{array}{c}\text { detuvieron 'si sono arrestate' } \\
\text { retomaron 'si sono riprese' }\end{array}$ & 5 studenti & 5 studenti \\
\hline $\begin{array}{c}\text { caras de miedo 'volti spaventati' } \\
\text { alrededor 'circa' }\end{array}$ & 3 studenti & 3 studenti \\
\hline $\begin{array}{c}\text { derrumbados 'crollati' } \\
\text { dicho 'detto' } \\
\text { tuvo 'ha avuto' } \\
\text { volvió 'è tornato' }\end{array}$ & 2 studenti & 2 studenti \\
\hline
\end{tabular}

Tabella 6. Le parole dichiarate difficili dagli studenti

Come prevedibile, sono percepite come problematiche le parole non trasparenti fra spagnolo e italiano, di cui non esistono né corrispondenti né falsi amici nelle due lingue, come detuvieron 'si sono arrestate' o alrededor 'intorno, circa', appartenenti a differenti classi del discorso, quali, ad esempio, i nomi (caras 'volti'), i verbi (detuvieron 'si sono arrestate'), gli aggettivi (derrumbados 'crollati') e gli avverbi (alrededor 'intorno, circa').

\subsubsection{Accuratezza morfologica}

Per misurare l'accuratezza morfologica delle trasposizioni degli studenti è stata effettuata una seconda tokenizzazione, in cui sono state escluse le parole morfologicamente invariabili del testo stimolo, in quanto prive di codifiche morfologiche da analizzare, quali nomi propri, nomi che indicano numeri, avverbi, congiunzioni, preposizioni semplici ${ }^{17}$. Tale tokenizzazione ha portato ad individuare 327 tokens significativi a livello morfologico, la cui accuratezza nei testi trasposti in L1 è stata valutata secondo la seguente scala:

a. 1 per le trasposizioni morfologicamente corrette, come il sostantivo alarma trasposto come "allarme", correttamente al singolare, nell'esempio seguente:

(8) St1. L'allarme è suonato quasi un minuto prima ( $<$ La alarma sonó casi un minuto antes);

b. 0.5 per le trasposizioni morfologicamente scorrette, come il sostantivo alarma trasposto come "allarmi", scorrettamente al plurale, nell'esempio seguente:

17 A differenza delle preposizioni semplici, la preposizione articolata del 'del', che codifica morfologicamente il tratto del genere e del numero, è stata inclusa nella tokenizzazione e nelle analisi. 
(9) St2. Gli allarmi sono suonati un minuto prima (< La alarma sonó casi un minuto antes);

c. 0 per le trasposizioni omesse.

Come illustrato nella Tabella 7, la maggior differenza nell'accuratezza morfologica fra i due gruppi risiede nel numero di trasposizioni omesse, che nel gruppo B è il triplo rispetto al gruppo A, analogamente a quanto emerso per l'accuratezza lessicale a causa delle difficoltà a completare la trasposizione (cfr. 4.2). Per le trasposizioni corrette, il gruppo A supera leggermente il gruppo B, mentre il numero di trasposizioni scorrette è simile nei due sottocampioni.

\begin{tabular}{|l|c|c|}
\hline & Gruppo A & Gruppo B \\
\hline Trasposizioni corrette & 289.50 & 228.90 \\
\hline Trasposizioni scorrette & 5.70 & 7.50 \\
\hline Trasposizioni omesse & 31.80 & 90.60 \\
\hline
\end{tabular}

Tabella 7. Tipologia di trasposizioni in L1 (media per gruppo)

Nella Tabella 8, che riporta l'accuratezza morfologica media della trasposizione in L1, si nota un vantaggio del $18.4 \%$ degli studenti di lingue, una percentuale quasi identica a quella della distanza fra i gruppi nell'accuratezza lessicale $(18.5 \%$, cfr. Tabella 5).

\begin{tabular}{|l|c|c|}
\hline & Gruppo A & Gruppo B \\
\hline Accuratezza morfologica & 292.35 & 232.05 \\
\hline
\end{tabular}

Tabella 8. Accuratezza morfologica della trasposizione in L1 (media per gruppo)

\subsubsection{Accuratezza sintattica}

Per misurare l'accuratezza sintattica delle trasposizioni in L1 degli studenti, i testi sono stati tokenizzati a livello di frase, contando le forme verbali (57), e i tokens sintattici sono stati valutati secondo la seguente scala:

a. 1 per le trasposizioni sintatticamente corrette, come l'esempio seguente:

(10) St4. la stessa zona dove lo scorso 7 settembre un terremoto di magnitudo 8,2 , il più forte dal 1932, ha causato 98 morti $(<$ la misma zona donde el 7 de septiembre pasado un terremoto de 8,2, el más poderoso desde 1932, causara 98 muertos)

b. 0.5 per le trasposizioni sintatticamente scorrette, come l'esempio seguente:

(11) St3. la stessa zona dove il 7 settembre si era verificato un terremoto di magnitudine 8,2 , il più forte dal 1932, che aveva causato 98 morti. (< la misma zona donde el 7 de septiembre pasado un terremoto de 8,2, el más poderoso desde 1932, causara 98 muertos) 
c. 0 per le trasposizioni omesse.

Come riportato nella Tabella 9, il gruppo A traspone in L1 correttamente un numero superiore di frasi rispetto al gruppo B e omette la trasposizione meno di un terzo delle volte, mentre la frequenza delle trasposizioni scorrette è simile fra i due sottocampioni.

\begin{tabular}{|l|c|c|}
\hline & Gruppo A & Gruppo B \\
\hline Trasposizioni corrette & 51.60 & 40.50 \\
\hline Trasposizioni scorrette & 0.10 & 0.20 \\
\hline Trasposizioni omesse & 5.30 & 16.30 \\
\hline
\end{tabular}

Tabella 9. Tipologia di trasposizioni in L1 (media per gruppo)

Le trasposizioni scorrette sono molto rare in entrambi i gruppi, in linea con i risultati di precedenti ricerche sull'intercomprensione fra le lingue romanze che sottolineano come il livello sintattico non crei particolari problemi di comprensione fra lingue affini (Bonvino et al. 2011). L'errore riscontrato nei dati ha interessato il periodo riportato in (10) e (11) (cfr. Appendice), lungo e sintatticamente complesso, in cui uno studente di lingue e due studenti di discipline non linguistiche hanno trasposto l'aggettivo pasado 'scorso', riferito al sostantivo septiembre ('settembre'), con una forma verbale ("si era verificato") e di conseguenza hanno modificato la struttura sintattica dell'intera frase, aggiungendo una proposizione relativa ("che aveva causato 98 morti”) e un ulteriore livello di dipendenza sintattica (11).

Come mostrato nella Tabella 10, l'accuratezza sintattica media delle trasposizioni in L1 degli studenti di lingue supera del 19.4\% quella degli studenti di discipline non linguistiche, in misura simile alla differenza relativa all'accuratezza lessicale $(18.5 \%)$ e all'accuratezza morfologica (18.4\%). Anche sul piano sintattico, nel gruppo B la distanza dal valore massimo (57) è dovuta all'alto numero di trasposizioni omesse (Tabella 9), per le ragioni già evidenziate in 4.2 .

\begin{tabular}{|l|c|c|}
\hline & Gruppo A & Gruppo B \\
\hline Accuratezza sintattica & 51.65 & 40.60 \\
\hline
\end{tabular}

Tabella 10. Accuratezza sintattica della trasposizione in L1 (media per gruppo)

\subsection{Strategie di comprensione}

Gli studenti di lingue e gli studenti di discipline non linguistiche hanno indicato le stesse strategie di comprensione (Tabella 11).

\begin{tabular}{|l|c|c|}
\hline & Gruppo A & Gruppo B \\
\hline Inferenza dal contesto & $10(75 \%)$ & $7(70 \%)$ \\
\hline Analogia con altre lingue & $2(8 \%)$ & $1(10 \%)$ \\
\hline Nessuna risposta & $1(17 \%)$ & $2(20 \%)$ \\
\hline Totale delle risposte & $12(100 \%)$ & $8(100 \%)$ \\
\hline
\end{tabular}

Tabella 11. Le strategie di comprensione autodichiarate dagli studenti 
Una differenza fra i due gruppi risiede nel numero totale di risposte fornite: gli studenti di lingue hanno indicato tutti almeno una strategia di comprensione e in due casi hanno specificato le strategie adoperate per più parole del testo, mentre tre studenti di discipline non linguistiche non hanno indicato alcuna strategia e solo uno fra coloro che hanno risposto ha esplicitato più di una strategia. Vi sono, invece, analogie fra i due sottocampioni per il tipo e la frequenza di verbalizzazioni fornite: la strategia più spesso dichiarata riguarda l'inferenza dal contesto (rispettivamente il $75 \%$ e il $70 \%$ delle risposte), seguita dal confronto interlinguistico alla ricerca di analogie fra la lingua target e le altre lingue note $(8 \%$ e $10 \%)$. Nonostante la maggior familiarità con i manuali di lingua e grammatica degli studenti di lingue, anche la profondità dell'argomentazione e l'uso di terminologia grammaticale tecnica risulta molto simile fra i due gruppi, come esemplificato dalle seguenti verbalizzazioni scritte da studenti di lingue in (12) e (14) e studenti di discipline non linguistiche in (13) e (15).

Inferenza dal contesto:

(12) St2 (lingue). Ho capito che sur significava 'sud' perché poco prima c'è la parola centro, che fa intendere che si sta parlando di una posizione geografica.

(13) St19 (spettacolo). Ho provato a dedurne il significato dal contesto.

Analogia con altre lingue:

(14) St2 (lingue). In parte ho capito il significato cercando di sfruttare le parole simili all'italiano.

(15) St16 (ingegneria). Detección: per la traduzione mi sono lasciata guidare dalla somiglianza con la parola inglese detection.

Per quanto riguarda la ricerca di analogie con altre lingue conosciute, è particolarmente interessante che gli studenti dichiarino di aver fatto riferimento sia alla L1 (14), sia ad una lingua non strettamente imparentata allo spagnolo come l'inglese (15), dimostrando di aver operato un confronto interlinguistico sulle lingue dell'intero repertorio personale.

Oltre alle dichiarazioni scritte, la correzione collettiva orale ha offerto ulteriori dati su quali elementi lessicali, morfologici e sintattici sono risultati utili per la comprensione, quali difficili e su quali strategie di comprensione sono state messe in atto. Nella discussione si sono realizzati casi di correzione fra pari, guidati non dalla ricercatrice, che conduceva l'esperimento con il ruolo di facilitatrice piuttosto che di docente, ma dagli altri partecipanti, che hanno collaborato molto fra loro per individuare la soluzione corretta. La sequenza interazionale riportata in (16) offre un esempio significativo di tale co-costruzione fra pari.

(16) St7. E poi in effetti bisogna aspettarsi altre repliche perché comunque possono arrivare fino ad otto queste repliche cioè ogni sisma si porta fino ad otto repliche appresso e quindi bisogna aspettarsi altri terremoti e scosse più violente.

St10. Ma dove le avete lette 'ste cose? 
St4. No, de uno a otro è 'dall'uno all'altro'!

Tutti: Ah, ecco! [Risate]

St4. los otros 'gli altri'! Vuagliù, un po' di Narcos!

Tutti: [Risate]

La sequenza interazionale riportata testimonia come gli studenti abbiano vissuto l'esperimento con coinvolgimento e rilassatezza, adoperando la varietà colloquiale ("ste cose"), elementi dialettali (vuagliù 'ragazzi') e facendo riferimento ad una serie tv ben nota (Narcos) come chiave per l'individuazione della risposta corretta (otro 'altro' non 'otto'). Proprio il riferimento alla serie tv è stato molto efficace tanto a livello comunicativo, suscitando una risata generale, quanto sul piano metacognitivo, dal momento che ha fornito un consiglio chiaro e concreto, utile per far capire l'errore ai compagni, e ha sottolineato l'importanza di riflettere su tutte le risorse linguistiche, anche quelle adoperate dai mass media o dai social networks, per la ricostruzione del significato del testo.

Sull'utilità dell'ascolto del testo letto da un parlante nativo dopo la prima lettura individuale ugualmente emergono analogie fra i due gruppi. In entrambi, per il $40 \%$ degli studenti l'input orale è stato di aiuto, mentre per il restante $60 \%$ l'ascolto non ha dato alcun supporto alla comprensione del testo scritto (Tabella 12).

\begin{tabular}{|l|c|c|}
\hline & Gruppo A & Gruppo B \\
\hline Utile & $4(40 \%)$ & $4(40 \%)$ \\
\hline Non utile & $6(60 \%)$ & $6(60 \%)$ \\
\hline
\end{tabular}

Tabella 12. Valutazione dell'utilità dell'ascolto

Le valutazioni positive sono state accompagnate da argomentazioni che hanno indicato il tipo di vantaggio prodotto dall'ascolto. Per entrambi i gruppi, l'input orale è stato di aiuto per cogliere il senso generale del testo, facendo focalizzare gli studenti su unità testuali ampie anziché su singole parole, per svelare somiglianze inaspettate fra spagnolo e italiano e per comprendere la struttura sintattica del testo, grazie alle pause che sottolineano i confini delle frasi. Al contrario, fra gli studenti che hanno giudicato non utile l'ascolto solo uno ha fornito una motivazione, dichiarando di aver percepito l'input orale come eccessivamente veloce (Tabella 13).

\begin{tabular}{|l|c|c|c|c|c|}
\hline \multicolumn{1}{|c|}{ Utile } & Gruppo A & Gruppo B & Non utile & Gruppo A & Gruppo B \\
\hline $\begin{array}{l}\text { Per cogliere il significato } \\
\text { generale del testo }\end{array}$ & $1(20 \%)$ & $2(50 \%)$ & $\begin{array}{c}\text { Eloquio } \\
\text { troppo } \\
\text { veloce }\end{array}$ & 0 & $1(10 \%)$ \\
\hline $\begin{array}{l}\text { Per riconoscere analogie } \\
\text { con altre lingue }\end{array}$ & $2(40 \%)$ & $1(25 \%)$ & & & \\
\hline $\begin{array}{l}\text { Per capire la sintassi del } \\
\text { testo }\end{array}$ & $2(40 \%)$ & 0 & & & \\
\hline $\begin{array}{l}\text { Per capire gli accenti } \\
\text { delle parole }\end{array}$ & 0 & $1(25 \%)$ & & & \\
\hline
\end{tabular}




\begin{tabular}{|l|c|c|c|c|c|}
\hline Nessuna motivazione & 0 & 0 & $\begin{array}{c}\text { Nessuna } \\
\text { motivazione }\end{array}$ & $10(100 \%)$ & $9(90 \%)$ \\
\hline
\end{tabular}

Tabella 13. Motivazioni dell'utilità dell'ascolto

\section{Osservazioni conclusive}

La ricerca conferma la buona competenza di intercomprensione dello spagnolo da parte di italofoni messa in evidenza dalla letteratura (Calvi 2001, 2004a, 2004b; Bailini 2016; Cortés Velásquez 2016; Di Gesù 2016). Gli studenti di lingue e gli studenti di discipline non linguistiche mostrano competenze analoghe nella comprensione del testo e nelle strategie di comprensione autodichiarate dai partecipanti, a conferma che l'intercomprensione fra lingue affini è un fenomeno di comunicazione plurilingue naturale e spontaneo, che si verifica anche in parlanti che non hanno né un repertorio linguistico molto ricco, né una competenza metalinguistica raffinata dallo studio specifico delle lingue. Negli studenti di discipline non linguistiche, la competenza linguistica è meno approfondita e la competenza metalinguistica è meno allenata e sviluppata, data la scarsa consuetudine con attività di riflessione sulle lingue, esercizi traduttivi e grammaticali, consultazione di manuali di grammatica, dizionari e testi in una lingua diversa dalla L1. Eppure la loro capacità di comprendere un testo in una lingua non nota come lo spagnolo non è inferiore a quella degli studenti di lingue sul piano della comprensione del contenuto e della verbalizzazione delle strategie di comprensione impiegate per accedere al significato. Entrambi i gruppi si mostrano in grado di comprendere il testo a livello globale, analitico e inferenziale e di esplicitare i processi messi in atto per risolvere il compito con argomentazioni simili. Gli studenti di lingue mostrano una migliore accuratezza lessicale, morfologica e sintattica nella trasposizione in L1 ma tale risultato è strettamente legato al fatto che quasi la metà degli studenti di discipline non linguistiche non è riuscita a completare la trasposizione in L1 nel limite di tempo assegnato, evidenziando difficoltà nell'analisi puntuale del testo spagnolo e nella stesura del testo italiano, non nell'accesso al significato del testo spagnolo. In sintesi, le analisi mostrano che la comprensione del contenuto si raggiunge anche nei parlanti che non dispongono di un ricco repertorio plurilingue e di una competenza metalinguistica maturata approfonditamente tramite lo studio delle lingue. Le differenze rispetto ai parlanti con repertorio plurilingue e competenza metalinguistica più sviluppata riguardano piuttosto i tempi necessari per la trasposizione in L1 del testo.

L'attenzione mostrata dagli studenti di discipline non linguistiche nelle domande conclusive e nella correzione orale collettiva conferma che non sono stati demotivati dalla difficoltà del compito di trasposizione e che hanno continuato a partecipare alle attività in modo attivo. Tale spinta motivazionale, insieme ai risultati positivi nella comprensione del contenuto e nella verbalizzazione delle difficoltà e strategie adottate, rimarca l'efficacia di attività di intercomprensione all'interno dei percorsi glottodidattici, soprattutto nelle fasi iniziali dell'acquisizione. L'intercomprensione può avere la funzione di stimolare la curiosità verso una nuova lingua, facendo toccare con mano la possibilità di accedere fin da subito ad una parte del significato di testi in lingue sconosciute sulla base delle risorse già a disposizione, come la naturale capacità di osservazione e analisi metalinguistica e la conoscenza di alme- 
no una lingua, la propria L1. Quante più lingue, anche conosciute in modo limitato o considerate di basso prestigio come i dialetti, rientrano nel repertorio linguistico personale, tanto più è profonda la capacità di intercomprensione, che così ricopre anche un importante ruolo di promozione del plurilinguismo. Le attività di intercomprensione sono in grado di aumentare la fiducia dei parlanti nelle loro capacità e la soddisfazione ad acquisire nuove conoscenze linguistiche (Tombolini 2018). L'intercomprensione infatti promuove un atteggiamento positivo e rilassato allo studio delle lingue, incrementando l'autostima dell'apprendente e mantenendo basso il filtro affettivo (Krashen 1981), costituito dalla paura di sbagliare e dall'ansia sulle proprie prestazioni linguistiche. L'apprendente ha diritto all'approssimazione, considerata funzionale ad un successivo approfondimento della conoscenza (Bonvino / Cortés Velásquez 2016a; Bonvino / Jamet 2016). La comprensione del significato è raggiunta per tappe, in un'ottica di problem solving autonomo e collaborativo fra pari, in cui l'apprendente è al centro dell'azione didattica e il docente ha il ruolo di facilitatore. Le fasi di lavoro autonomo degli apprendenti vanno integrate con momenti di confronto con il docente, che individui i problemi intercomprensivi e proponga percorsi per raggiungere le soluzioni in maniera induttiva, sfruttando le risorse linguistiche e metalinguistiche degli studenti.

Queste considerazioni e i risultati qui presentati, che documentano il successo delle attività di intercomprensione anche per studenti universitari che non hanno approfondite competenze linguistiche e metalinguistiche, suggeriscono l'impiego dell'intercomprensione, oltre che negli insegnamenti dei corsi di studio in lingue straniere, nei corsi di lingue per non specialisti, quali quelli organizzati dai centri linguistici di ateneo o dai dipartimenti di lingue straniere per gli studenti universitari di qualsiasi disciplina, che sono mirati ad un insegnamento di base di una lingua e cultura straniera, nell'ottica di attirare più studenti verso l'apprendimento di lingue affini ed educare una comunità di parlanti plurilingui più vasta possibile.

\section{Appendice}

\section{Un nuevo temblor sacude el centro y sur de México}

El seísmo magnitud 6,1 en la escala de Richter se trata de una réplica del que se produjo el pasado 7 de septiembre. Dos personas han muerto en la capital a causa de un infarto

\section{EL PAÍS, México 24 SEP 2017 - 17:27}

Un seísmo de magnitud 6,1 en la escala de Richter volvió a sacudir este sábado por la mañana el centro y sur de México y ha desatado los peores temores de los que vivieron cuatro días antes el poderoso terremoto de 7,1 que ha causado hasta el momento alrededor de 300 víctimas mortales. Las labores de rescate en la capital se detuvieron unos minutos, según informaron las autoridades, y se retomaron poco después. El temblor ha sido una réplica del que se produjo el 7 de septiembre, según han confirmado las autoridades, y ha debilitado algunas construcciones en Oaxaca que ya estaban afectadas por el seísmo anterior que dejó 98 muertos. Dos personas han fallecido a causa de un infarto en Ciudad de México. 
El movimiento telúrico se registró a las 7.53 hora local y su epicentro se localizó en el sureño estado de Oaxaca, la misma zona donde el 7 de septiembre pasado un terremoto de 8,2, el más poderoso desde 1932, causara 98 muertos. "Al momento, las mayores afectaciones son el puente de Ixtaltepec [Oaxaca], que deberá reconstruirse, y estructuras con daños previos que colapsaron", ha informado el presidente mexicano, Enrique Peña Nieto.

La alarma sonó casi un minuto antes de que se pudiera sentir el temblor en Ciudad de México, lo que permitió a muchos ciudadanos abandonar sus casas y refugiarse en la calle. La escena se repetía, las caras de miedo, el "ha vuelto a pasar". Los 38 edificios derrumbados en la capital los llevan tatuados en la piel y quedarse dentro no es una opción.

En Ciudad de México se sintió una "percepción ligera y por el momento no reportan afectaciones, continúa el monitoreo", señaló en Twitter el coordinador nacional de Protección Civil, Luis Felipe Puente, quien dijo estar en comunicación con los estados de Chiapas y Oaxaca.

"Pedimos a toda la población mantener la calma", indicó a su vez la Presidencia. El director del Centro Nacional de Prevención de Desastres (Cenapred), Carlos Valdés, confirmó que el sismo fue una réplica del ocurrido el 7 de septiembre. Por la "magnitud" que tuvo dicho terremoto, señaló, "puede generar réplicas como la que hemos visto el día de hoy".

"Cada sismo se comporta de forma diferente. El número de réplicas varía de uno a otro. La instrumentación se ha incrementado y hoy es fácil ver sismos pequeñitos y réplicas pequeñitas. Se debe considerar que el movimiento del 7 de septiembre está produciendo muchas réplicas y que tenemos una mejor capacidad de detección", explicó Xyoli Pérez Campos, directora del Servicio Sismológico Nacional (SSN). Según los estudios de los expertos, hasta el día de hoy se han registrado nada menos que 4.287 réplicas del movimiento de hace dos semanas.

\section{Referencias bibliográficas}

Andersen, Roger (1983): «Transfer to somewhere», in Suzanne Gass, Larry Selinker (eds.), Language transfer in language learning, Rowley, Newbury House, pp. 177201.

Andorno, Cecilia / Valentini, Ada / Grassi, Roberta (2017): Verso una nuova lingua. Capire l'acquisizione di L2, Torino, UTET.

Aslanov, Cyril (2016): «La lingua franca fra ieri ed oggi», in Elisabetta Bonvino / Marie-Christine Jamet (a c. di), Intercomprensione: lingue, processi e percorsi, Venezia, Ca' Foscari, pp. 29-42.

Bailini, Sonia (2016): La interlengua de lenguas afines. El español de los italianos, el italiano de los españoles, Milano, LED.

Balboni, Paolo (2018): Fare educazione linguistica. Insegnare italiano, lingue straniere e classiche, Torino, UTET.

Benucci, Antonella (a c. di) (2005): Le Lingue romanze. Una guida per l'intercomprensione, Torino, UTET.

Benucci, Antonella (2015): L'intercomprensione: il contributo italiano, Torino, UTET.

Berretta, Monica (1984): «La competenza linguistica nella scuola di base», AA.Vv, L'educa- 
zione linguistica dalla scuola di base al biennio della superiore. Atti de convengo CIDI LEND (Viareggio, 3-6 marzo 1983), Milano, Mondadori, pp. 148-161.

Bettoni, Camilla (2001): Imparare un'altra lingua: lezioni di linguistica applicata, Roma, Laterza.

Bialystok, Ellen (1988): «Levels of Bilingualism and Levels of Linguistic Awareness», Developmental Psychology, 24/4, pp. 560-567.

Blanche-Benveniste, Claire / Valli, André / Mota, Antónia/ Simone, Raffaele / Bonvino, Elisabetta / Uzcanga De Vivar, Isabel (1997): EuRom4, metodo di insegnamento simultaneo delle lingue romanze, Firenze, La Nuova Italia.

Blanche-Benveniste, Claire / Valli, André (a c. di) (1997): L'Intercompréhension: le cas des langues romanes. Le français dans le monde. Recherches et applications (numero monografico).

Bonvino, Elisabetta (2012): «Lo sviluppo delle abilità di lettura nell'ottica dell'intercomprensione», in Elisabetta Bonvino, Eleonora Luzi, Anna Rita Tamponi (a c. di), (Far) apprendere, usare e certificare una lingua straniera, Roma, Bonacci, pp. 139-152.

Bonvino, Elisabetta / Caddéo, Sandrine / Vilaginés Serra, Eulàlia / Pippa, Salvador (2011): EuRom5: leggere e capire 5 lingue romanze, Milano, Hoepli.

Bonvino, Elisabetta / Cortés Velásquez, Diego (2016a): «Il lettore plurilingue», Lend, 4, pp. 111-130.

Bonvino, Elisabetta / Cortés Velásquez, Diego (2016b): «La intercomprensión y la enseñanza de las lenguas romances : propuesta para una formación metacognitiva a la comprensión oral», in Éva Buchi, Jean-Paul Chauveau, Jean-Marie Pierrel (a c. di), Actes du XXVIIe Congrès international de linguistique et de philologie romanes, Strasbourg, Éditions de linguistique et de philologie, pp. 1283-1296.

Bonvino, Elisabetta / Jamet, Marie-Christine (a c. di) (2016): Intercomprensione: lingue, processi e percorsi, Venezia, Edizioni Ca' Foscari.

Calvi, Maria Vittoria (1995): Didattica di lingue affini: spagnolo e italiano, Milano, Guerini.

Calvi, Maria Vittoria (1999): «Los neologismos en la enseñanza del español a extranjeros», in Maria Vittoria Calvi, Félix San Vicente (a c. di), Palabras de acá y de allá, Viareggio, Baroni, pp. 49-60.

Calvi, Maria Vittoria (2001): «Comprendere un testo spagnolo: trasparenze, opacità e falsi amici», in Antonella Cancellier, Rernata Londero (a c. di), Italiano e spagnolo a contatto. Atti del XIX Convegno AISPI, Roma, Unipress, pp. 55-68.

Calvi, Maria Vittoria (2004a): "Apprendimento del lessico di lingue affini», Cuadernos de Filología Italiana, 11, pp. 61-71.

Calvi, Maria Vittoria (2004b): «Aprendizaje de lenguas afines, español e italiano», redELE, $1,<$ http://www.educacion.es/redele/revista1/calvi.shtml $>$.

Camps, Anna / Milian, Marta (a c. di) (1999): Metalinguistic Activity in Learning to Write, Amsterdam, Amsterdam University Press.

Candelier, Michel / Camilleri-Grima, Antoinette / Castellotti, Véronique / de Pietro, Jean-François / Lörincz, Ildikó / Meissner, Franz-Joseph / Schröder-Sura, Anna / Noguerol, Artur (2007): Le CARAP - Un cadre de référence pour les approches plurielles des langues et des cultures. Compétences et ressources, Strasbourg/Graz, Conseil de l'Europe.

Cappelli, Deborah (2019): «The importance of the transfer in Italian - Spanish learning», Quaderni di Linguistica e Studi Orientali, 5, pp. 345-366.

Compagno, Giuseppa / Floriana, Di Gesù (2007): «Le strategie d'intercomprensione spagnolo/italiano nell'analisi contrastiva delle canzoni», in Lorenzo Blini, Maria Vittoria Calvi, Antonella Cancellier (a c. di), Lingüística contrastiva entre el italiano y las lenguas 
ibéricas / Linguistica contrastiva tra italiano e lingue iberiche, Madrid, Instituto Cervantes, pp. 131-144.

Consiglio d'Europa (2001): Common European Framework of Reference for Languages. Learning, Teaching, Assessment, Strasburgo, Consiglio d'Europa.

Cortés Velásquez, Diego (2016): «La trasparenza lessicale nella comprensione orale. Analisi di un corpus di dati sull'ascolto dello spagnolo e il portoghese», in Elisabetta Bonvino, Marie-Christine Jamet (a c. di), Intercomprensione: lingue, processi e percorsi, Venezia, Edizioni Ca' Foscari, pp. 81-111.

Dabène, Louise (1975): «L'enseignement de l'espagnol aux francophones (pour une didactique des langues voisines)», Langages, 39, p. 51-64.

Dabène, Louise / Degache, Christian (a c. di) (1996): Comprendre les langues voisines. Études de Linguistique Appliquée, 104 (numero monografico).

De Benedetti, Andrea (2006): «Liscio come l'aceite. Errori di interferenza (e non) nell'apprendimento dell'italiano L2 in parlanti ispanofoni», in Franca Bosc, Carla Marello, Silvana Mosca (a c. di), Saperi per insegnare, Torino, Loescher, pp. 205-217.

De Carlo, Maddalena (a c. di) (2011): Intercomprensione e educazione al plurilinguismo, Porto Sant'Elpidio, Wizarts.

Degache, Christian / Masperi, Monica (1998): «La communication plurilingue en toile de fond de l'entraînement à la compréhension des langues romanes»), in Jacqueline Billiez (a c. di), De la didactique des langues à la didactique du plurilinguisme. Hommage à Louise Dabène, Grenoble, Lidilem, pp. 361-376.

Della Putta, Paolo (2016): «The effects of Textual Enhancement on the acquisition of two non-parallel Italian features by Spanish-Speaking learners of Italian», Studies in Second Language Acquisition, 38, pp. 217-238.

Della Putta, Paolo (2017): Apprendimento e disapprendimento fra spagnolo e italiano. Uno studio bidirezionale sull'efficacia di una tecnica di Focus on Form, Roma, Aracne.

Di Gesù, Floriana (2016): Linguistica contrastivo-percettiva di lingue tipologicamente affini: italiano e spagnolo, Palermo, Palermo University Press.

Ellis, Rod (2004): «The definition and measurement of explicit knowledge», Language Learning, 54, pp- 227-275.

Ellis, Rod (2005): «Measuring implicit and explicit knowledge of a second language: A psychometric study», Studies in Second Language Acquisition, 27, pp. 141-172.

Ellis, Rod (2006): «Modelling learning difficulty and second language proficiency: The differential contributions of implicit and explicit knowledge», Applied Linguistics, 27, pp. 431-463.

Ferrario, Gloria (2009): «L'italiano degli immigrati ispanofoni. L'influenza della Lingua 1 nell'apprendimento di lingue affini», Italiano LinguaDue, 1, pp. 314-340.

García Castanyer, Maria Teresa / Vilaginés Serra, Eulàlia (2010): «Romanicaintercom et le projet d'intercompréhension en 8 langues romanes», Synergies Europe, 5, pp. 53-62.

Gass, Susan / Selinker, Larry (a c. di) (1983): Language transfer in language learning, Rowley, Newbury House.

Jamet, Marie Christine (2010): «Intercomprensione, Quadro comune europeo di riferimento per le lingue, Quadro di riferimento per gli approcci plurilingui e valutazione», Synergies Europe, 5, pp. 75-98.

Jarvis, Scott / Pavlenko, Aneta (2008): Crosslinguistic Influence in Language and Cognition, New York, Routledge.

Klein, Horst G. / Stegmann, Tilbert D. (1999): EuroComRom. Die sieben Siebe. Romanische Sprachen sofort lesen können, Aachen, Shaker. 
Koessler, Maxime / Derocquigny, Jules (1928): Les faux amis ou les trahisons du vocabulaire anglais, Paris, Librairie Vuibert.

Krashen, Stephen D. (1981): Second Language Acquisition and Second Language Learning, Oxford, Pergamon.

Laufer, Batia (1997): «The lexical plight in second language reading. Words you don't know, words you think you know, and words you can't guess», in James Coady, Thomas Huckin (eds.), Second Language Vocabulary Acquisition. A Rationale for Pedagogy, Cambridge, Cambridge University Press, pp. 20-34.

Lo Duca, Maria Giuseppina (2003): Lingua italiana ed educazione linguistica, Roma, Carocci.

Lo Duca, Maria Giuseppina (2004): Esperimenti grammaticali. Riflessioni e proposte sull'insegnamento della grammatica dell'italiano, Roma, Carocci.

Lo Duca, Maria Giuseppina (2012): «Tra competenza metalinguistica e curricolo grammaticale», in Giuliano Bernini, Cristina Lavinio, Ada Valentini, Miriam Voghera (a c. di), Atti dell'11 ${ }^{\circ}$ Congresso AItLA, Perugia, Guerra: 331-337.

Lumbelli, Lucia (2009): La comprensione come problema. Il punto di vista cognitivo, Bari, Laterza.

Napolitano, Francesca (2018): El potencial de la intercomprensión en la didáctica de lenguas: informe de una investigación en la Universidad de Salerno (tesi di laurea inédita), Salerno, Università di Salerno.

Odlin, Terence (1989): Language transfer. Crosslinguistic Influence in Language Learning, Cambridge, Cambridge University Press.

Pinto, Maria Antonietta (2015): The “MATEL” Project: Research Result, Roma, Sapienza Editrice.

Roehr, Karen (2007): «Metalinguistic knowledge and language ability in university-level L2 learners», Applied Linguistics, 29/2, pp. 173-199.

Rosi, Fabiana (2009): Learning Aspect in Italian L2: Corpus Annotation, Acquisitional Patterns, and Connectionist Modelling, Milano, Franco Angeli.

Rosi, Fabiana (2020a): «L'uso delle perifrasi aspettuali e modali per costruire la referenza in italiano e spagnolo L1 e LS», in Fernado Molina Castillo, Alicia López Márquez (eds.), Italiano y español. Estudios de traducción, lingüística contrastiva y didáctica, Bern, Peter Lang, pp. 391-403.

Rosi, Fabiana (2020b): «Comprendere i testi per lo studio in italiano L1 e L2: competenze e bisogni degli studenti universitari», Italiano LinguaDue, 12, pp. 143-158.

Schmid, Stefan (1994a): L'italiano degli spagnoli, Milano, Franco Angeli.

Schmid, Stefan (1994b): «Un modello di strategie di acquisizione per lingue comparate», in Anna Giacalone Ramat, Massimo Vedovelli (a c. di), Italiano lingua seconda / lingua straniera. Atti del XXVI Convegno internazionale di studi della Società di linguistica italiana, Roma, Bulzoni, pp. 61-79.

Simone, Raffaele (1997): «Langues romanes de toute l'Europe, unissez-vous!», in Claire Blanche-Benveniste, André Valli (a c. di), L'Intercompréhension: le cas des langues romanes. Le français dans le monde. Recherches et applications (numero monografico), pp. 25-33.

Tagliavini, Carlo (1947): «Spagnolo e italiano», Le lingue estere, 12, pp. 261-262, 275-276.

Tombolini, Annalisa (2018): «Intercomprensione orale tra lingue romanze. Cognizione ed emozione all'ascolto di suoni e di significati sperimentando nuovi percorsi didattici in uno studio di caso», in Carmel Mary Coonan, Ada Bier, Elena Ballarin (a c. di), La didattica delle lingue nel nuovo millennio Le sfide dell'internazionalizzazione, Venezia, Ca' Foscari, pp. 663-680. 
van Someren, Maarten W. / Barnard, Yvonne / Sandberg, Jacobijn (1994): The Think Aloud Method: A Practical Guide to Modelling Cognitive Processes, London, Academic Press. Vilaginés Serra, Eulàlia (2007): «El reto de la intercomprensión», in Dominque Bonnet, María José Chaves García, Nadia Duchêne (a c. di), Littérature, langages et arts: rencontre et création, Huelva, Servicio de Publicaciones de la Universidad de Huelva, CDRom.

Voghera, Miriam (2008): «Lingua e testi: verso una grammatica comune», Testi e Linguaggi, 2, pp. 9-17. 